\title{
Study on syndrome differentiation strategy of phlegm and blood stasis syndromes of coronary heart disease based on expert consultation on medical cases
}

\author{
Ren Liu ${ }^{1,2} \wedge$, Li-Jie Jiang ${ }^{2}$, Yan Yang ${ }^{2}$, Chuan-Chi Wang ${ }^{2}$, Xu Tong ${ }^{2}$, Wei-Ming Xu ${ }^{2}$, Meng Wu ${ }^{2}$, \\ Ke-Zhi Lu ${ }^{3}$, Jing-Qing $\mathrm{Hu}^{1,2}$
}

${ }^{1}$ Affiliated Hospital of Integrated Traditional Chinese and Western Medicine, Nanjing University of Chinese Medicine, Nanjing, China; ${ }^{2}$ Institute of Basic Theory for Chinese Medicine, China Academy of Chinese Medical Sciences, Beijing, China; ${ }^{3}$ School of Computer and Information Technology, Beijing Jiaotong University, Beijing, China

Contributions: (I) Conception and design: JQ Hu, LJ Jiang, WM Xu; (II) Administrative support: LJ Jiang, X Tong; (III) Provision of study materials or patients: CC Wang, R Liu, LJ Jiang; (IV) Collection and assembly of data: R Liu, LJ Jiang, Y Yang, KZ Lu; (V) Data analysis and interpretation: R Liu, LJ Jiang, KZ Lu, JQ Hu, Y Yang, WM Xu, M Wu, X Tong; (VI) Manuscript writing: All authors; (VII) Final approval of manuscript: All authors.

Correspondence to: Jing-Qing Hu. Affiliated Hospital of Integrated Traditional Chinese and Western Medicine, Nanjing University of Chinese Medicine, Nanjing, China; Institute of Basic Theory for Chinese Medicine, China Academy of Chinese Medical Sciences, Beijing, China. Email: gcp306@126.com.

Background It is becoming more and more important to judge whether patients with coronary heart
disease (CHD) have phlegm and blood stasis syndromes in the process of traditional Chinese medicine
(TCM) diagnosis and treatment of CHD. The syndrome differentiation strategy of phlegm and blood stasis
syndromes of CHD is still not standardized, and it is particularly necessary to make syndrome differentiation
simpler and more accurate.

Methods: Twenty-eight medical cases that met the criteria, comprising 10 ancient medical cases and 18 modern ones, were selected from the TCM literature, which were then analyzed by 57 experts via questionnaire. Statistical analysis of the data was mainly based on frequency analysis.

Results: (I) The average age of the 57 experts from 20 provinces was $48.9 \pm 8.5$ years; $89.5 \%$ were associate professor or above, and $75.4 \%$ of them worked at a tertiary hospital. (II) Consistency of expert consultation over medical cases: for the ancient medical cases, the diagnostic consistency rate of phlegm syndrome was $27 / 34(79.4 \%)$ and additional diagnosis rate of the blood stasis syndrome was 27/57 (47.4\%); for the modern medical cases, the consistency rate compared with the original diagnosis of phlegm syndrome was 54/80 (67.5\%) and that of blood stasis syndrome was 73/90 (81.1\%). (III) The top five experts' diagnostic basics of phlegm syndrome were oppression in the chest, slippery pulse, greasy fur, coughing of phlegm, and chest pain; the top five diagnostic basics of blood stasis syndrome were chest pain, dark tongue, oppression in chest, red tongue, and ecchymosis on tongue. (IV) In the questionnaire consultation on CHD phlegm-blood stasis syndrome cases, the diagnostic basis of "symptom or (and) tongue manifestation" accounted for 12/27 (44.4\%) of the diagnostic basics of phlegm syndrome and 28/38 (73.7\%) of that of blood stasis syndrome basis.

Conclusions: Modern Chinese medicine experts pay much attention to the diagnosis and treatment of CHD based on TCM pathology theories of phlegm and blood stasis. To collect and detect the patients' symptoms and tongue manifestation is an important strategy of the experts for CHD phlegm and blood stasis syndrome differentiation.

^ ORCID: 0000-0001-9960-7803. 
Keywords: Blood stasis syndrome; coronary heart disease (CHD); medical cases; phlegm syndrome; syndrome differentiation strategy

Submitted Jul 23, 2021. Accepted for publication Sep 15, 2021.

doi: 10.21037/apm-21-2332

View this article at: https://dx.doi.org/10.21037/apm-21-2332

\section{Introduction}

Coronary heart disease (CHD), also known as coronary artery disease, is caused by the buildup of coronary atherosclerotic plaque or coronary artery spasm, which may lead to coronary artery stenosis and blood supply limitation, and cause myocardial ischemia and hypoxia. In recent years, despite the widespread use of modern medical technologies, CHD is still one of the leading causes of death worldwide and a common cause of hospital admission $(1,2)$.

In China, besides applying modern medicine to treat CHD, traditional Chinese medicine (TCM) is also widely used. Syndrome differentiation is a crucial step in the process of TCM diagnosis and treatment. It is an important step to judge whether a patient has the syndrome of phlegm or blood stasis when differentiating the syndrome of CHD. According to TCM theories and clinical data, phlegm and blood stasis syndromes are major ones of CHD. The results of an epidemiological survey showed that in mainland China, $77.89 \%$ of the total incidence of CHD was blood stasis syndrome, while $43.97 \%$ was phlegm syndrome (3). According to a cross-sectional study of 11,383 cases, the proportion of phlegm syndrome and blood stasis syndrome has increased along with the occurrence and development of coronary heart disease (4). The reason is that the pathological process of phlegm and blood stasis is consistent with that of CHD. Large intake of high-fat foods and reduced exercise will aggravate the body's lipid deposition, elevate blood lipids and blood cell adhesion, leading to the occurrence and development of phlegm syndrome and blood stasis syndrome (5). Dan Shen Yin Decoction and Tao Hong Si Wu Decoction were both classical formulas to treat blood stasis syndrome with CHD, Gua Lou Xie Bai Ban Xia Decoction and Huang Lian Wen Dan Decoction were both classical formulas in treating CHD with phlegm syndrome (6).

Macrocosmic diagnostic is basic means of syndrome differentiation of phlegm and blood stasis in TCM for coronary heart disease. Its criteria for CHD with phlegm and blood stasis syndromes has been established, which could help to standardize the measurement and improve the level of TCM prevention and treatment of the syndromes of phlegm and blood stasis in CHD (7). The research of syndrome differentiation not only needs to develop the diagnostic criteria of syndrome, but also to inspire the way to use the criteria through the research of syndrome differentiation strategy (8). Syndrome differentiation strategy is regarded as a specific skill of individual doctors in clinical practice under the guidance of the general principles of syndrome differentiation (8). Development of the syndrome differentiation strategy to enhance the accuracy of clinical syndrome differentiation results is a key issue in clinical research of TCM. TCM experts are of many years of clinical experience, systematic and profound theoretical cognition, and high accuracy and skills in syndrome differentiation. There is a great clinical value in studying their strategy of syndrome differentiation. Therefore, this study invited the experts to diagnose the selected CHD medical cases, and analyzed the results of their diagnosis on phlegm syndrome and blood stasis syndrome and their diagnosis basis of syndrome diagnosis, so as to form the phlegm syndrome and blood stasis syndrome differentiation strategy of CHD. This study took the lead in the study of CHD syndrome differentiation strategy, which would provide a methodological inspiration for the future study of syndrome differentiation strategy, and a reference for the application of the diagnostic criteria of CHD with phlegm syndrome and blood stasis syndrome.

We present the following article in accordance with the SURGE reporting checklist (available at https://dx.doi. org/10.21037/apm-21-2332).

\section{Methods}

The CHD syndrome differentiation questionnaire was based on both ancient and modern TCM medical cases. TCM medical cases are important carriers of knowledge with regard to symptoms of the patients, treatment methods, prescriptions, herbs, as well as the notes about the cases by the original authors, which expound the doctors' 
thinking and experience of diagnosis and treatment.

The main research process was as follows: First, CHD medical cases were selected from all the medical cases of ancient and modern times, and the CHD syndrome differentiation questionnaire was constructed. Then the experts were invited to complete the questionnaire. Finally, the results were analyzed, and the strategy of the experts on syndrome differentiation of CHD with phlegm and blood stasis syndromes was summarized.

\section{Questionnaire production}

\section{Sources of the medical cases}

The sources of CHD cases included both ancient and modern TCM literature. CHD is a modern medical term, which is very close to the symptoms of xiong bi (literally means chest obstruction) in TCM. The main symptom of xiong bi is pain in the chest, or even pain involving the back and the patient cannot lie down to breathe. Therefore, xiong bi cases in the ancient TCM literature which have a high probability of being patients with CHD were selected, together with CHD cases, as the basic materials for this study. The cases of xiong bi were screened from the ancient TCM literature, such as Classified Case Records of Celebrated Physicians, Supplement to Classified Case Records of Celebrated Physicians, Guide to Clinical Practice with Medical Records, etc. Sources of CHD medical cases included articles and books that published the first and second batch of the national TCM masters' cases.

\section{Inclusion and exclusion criteria of the medical cases}

The inclusion criteria of ancient xiong bi medical cases: (I) the diagnosis of disease conformed to the diagnosis of xiong bi in Chinese Internal Medicine (9): (i) The main symptom of patients is chest pain, and they often suffer from suffocating pain in the precordial area, even pain in the left shoulder, back, throat, stomach, inside of left upper arm and teeth. The pain is recurrent and lasts from a few seconds to dozens of minutes, and can be relieved after rest or medication. (ii) Pain is often accompanied by palpitation, shortness of breath, spontaneous sweating, even wheezing and inability to lie down. In severe cases, the patients may feel intense pain, feeling cold in the limbs after perspiration, pale face, and blue or purple lips, scattered, faint, thin or undetectable pulse, and even sudden death. (iii) Chest pain is often seen in middle-aged or older patients, and often occurs after overwork, depression, anger, excessive drinking or change in climate. It can also occur without any inducement or activity. (II) The medical cases were complete, including four items of diagnostic information: diagnosis, treatment principles and methods, prescriptions and medicinals.

The exclusion criteria of ancient medical cases: (I) medical cases with main symptoms of exogenous disease, gastroenterological disease, gynecological disease, surgical disease combined with chest tightness, chest pain, or chest fullness; (II) medical cases with chest tightness, chest pain, or chest fullness during pregnancy.

The inclusion criteria of modern medical cases: (I) the disease diagnosis was consistent with the diagnosis of CHD, including patients in the stable or unstable stage and after percutaneous coronary intervention/coronary artery bypass grafting; (II) the clinical syndrome differentiation information was comprehensive, including the four items of diagnostic information: diagnosis, treatment principles and methods, prescriptions and medicinals.

The exclusion criteria of modern medical cases: the main complaint of the first visit or the purpose of medical treatment was not for CHD.

\section{Syndrome differentiation normalization of the medical cases}

The syndrome diagnosis of the ancient medical cases was formed through pathogenesis analysis, treatment method, prescription and notes. Original syndrome differentiation diagnosis was taken as the main basis for syndrome differentiation diagnosis of the modern medical cases, which also referred to their pathogenesis analysis, treatment methods, prescriptions and notes. After the diagnosis of syndrome was standardized, if the medical case had phlegm and blood stasis syndromes at the same time, the medical case was determined as phlegm-blood stasis syndrome.

\section{Selection of the medical cases}

According to the selection criteria described above, two researchers conducted the preliminary selection from Collection of the Medical Cases of Ancient Xiong Bi and Coronary Heart Disease by National Chinese Medicine Masters, and then the third researcher conducted the secondary selection. Finally, 28 medical cases were selected, comprising 10 ancient medical cases and 18 modern medical cases. There were 9 cases of phlegm-blood stasis syndrome, 9 cases of phlegm syndrome, 5 cases of blood stasis syndrome, and 5 cases of neither phlegm nor blood stasis syndrome.

All the diagnostic and treatment plans of the medical cases selected were effective, which can be used as the 
gold standards to assess others syndrome diagnoses. In particular, 18 modern medical cases showed the effect of the diagnosis and treatment plan, and the curative effect was satisfactory. After treatment, the chest pain was relieved or went away, the blood pressure was well controlled, the dosage of western medicine was reduced or stopped, the accompanying symptoms were alleviated or disappeared, and the tongue and pulse returned to normal.

\section{Form and content of the questionnaire}

The expert syndrome differentiation questionnaire were divided into four parts: the basic information about the expert, instruction for filling out questionnaire, three medical cases, and questions. Of the three medical cases, one was an ancient xiong bi medical case and two were CHD medical cases. At least one of the two CHD medical cases was a phlegm and blood stasis syndrome case. The medical cases included basic information of the patients, symptoms and signs, and the main results of modern medical examination. The experts surveyed were supposed to answer two questions. The first one was the syndrome diagnosis and syndrome diagnostic basis. Experts produced their syndrome diagnosis by selecting from 10 options without limiting the number of options that could be selected: phlegm, qi stagnation, yang deficiency, fire-heat, dampness, cold coagulation, qi deficiency, yin deficiency, blood stasis, and others. If the expert selected "others", they should describe the specific syndrome diagnosis. The experts also needed to write their syndrome diagnostic basis for the selected options. The second question was the treatment prescription for the cases. There was no similar questionnaire before. The questionnaire had been revised for three rounds in the research group to ensure the clarity of sentence expression and to meet the research purpose.

\section{Questionnaire survey}

\section{Selection of experts}

Based on the research objectives, the selection criteria of experts were as follows. (I) Professional status: mainly senior physician professional titles. (II) Specialty: TCM/integrated Chinese and western medicine physicians in cardiovascular and cerebrovascular disease. (III) Physician with more than 5 years' working experience. (IV) Institution: priority given to Grade III Level A hospital or research institution of TCM. (V) Willing to participate in the investigation and in completing the questionnaire.

The questionnaire survey was carried out in Beijing in
April 2018 and in Wuhan in May 2018, the experts were required to complete the questionnaire on the day of the survey with their knowledge of TCM theory and clinical experience.

This study did not carry out treatment intervention for any patient. The medical cases used are open and shared. The extracted information included only the judgment of the experts on the medical cases, without personal information such as contacts. Therefore, ethical review or the approval of the ethical review committee was not required for this study. But we paid great attention to protecting the rights and informed consent of respondents. The participants have given informed consent before taking part. The study was conducted in accordance with the Declaration of Helsinki (as revised in 2013). The experts were informed of the research purpose of the questionnaire, the data utilization plan and their rights and interests, and we have gained their consent to participate in the research. The first page of the questionnaire emphasized the content and data utilization plan of this study. The experts were reimbursed for reasonable transport and consultation costs.

\section{Data processing and statistical analysis}

Databases were established with Microsoft Excel and double-entry data input. If the syndrome differentiation result of an expert was in doubt, the treatment method and prescription information will be used as a reference. Terminology specification of symptoms was taken from the Differential Diagnosis of TCM Symptoms (Second Edition) (10) and Chinese Terms in Traditional Chinese Medicine and Pharmacy (11).

The results of syndrome differentiation diagnosis among the experts were compared and the frequency of a diagnostic basis for phlegm or blood stasis syndrome was counted. The occurrence rate of a syndrome refers to the ratio of the number of medical cases that at least one expert diagnosed the patient as having this syndrome to the total number of medical cases. The diagnostic consistency rate of a syndrome refers to the ratio of the number of experts whose syndrome diagnosis result was consistent with the medical case author's syndrome diagnosis to the number of experts who answered the same medical case. The additional syndrome diagnosis rate refers to the ratio of the number of experts who gave a syndrome diagnosis that was not found in the original syndrome diagnosis of the medical cases to the answered times of the case without this syndrome diagnosis. 
Table 2 Syndrome differentiation consistency between the original cases and experts

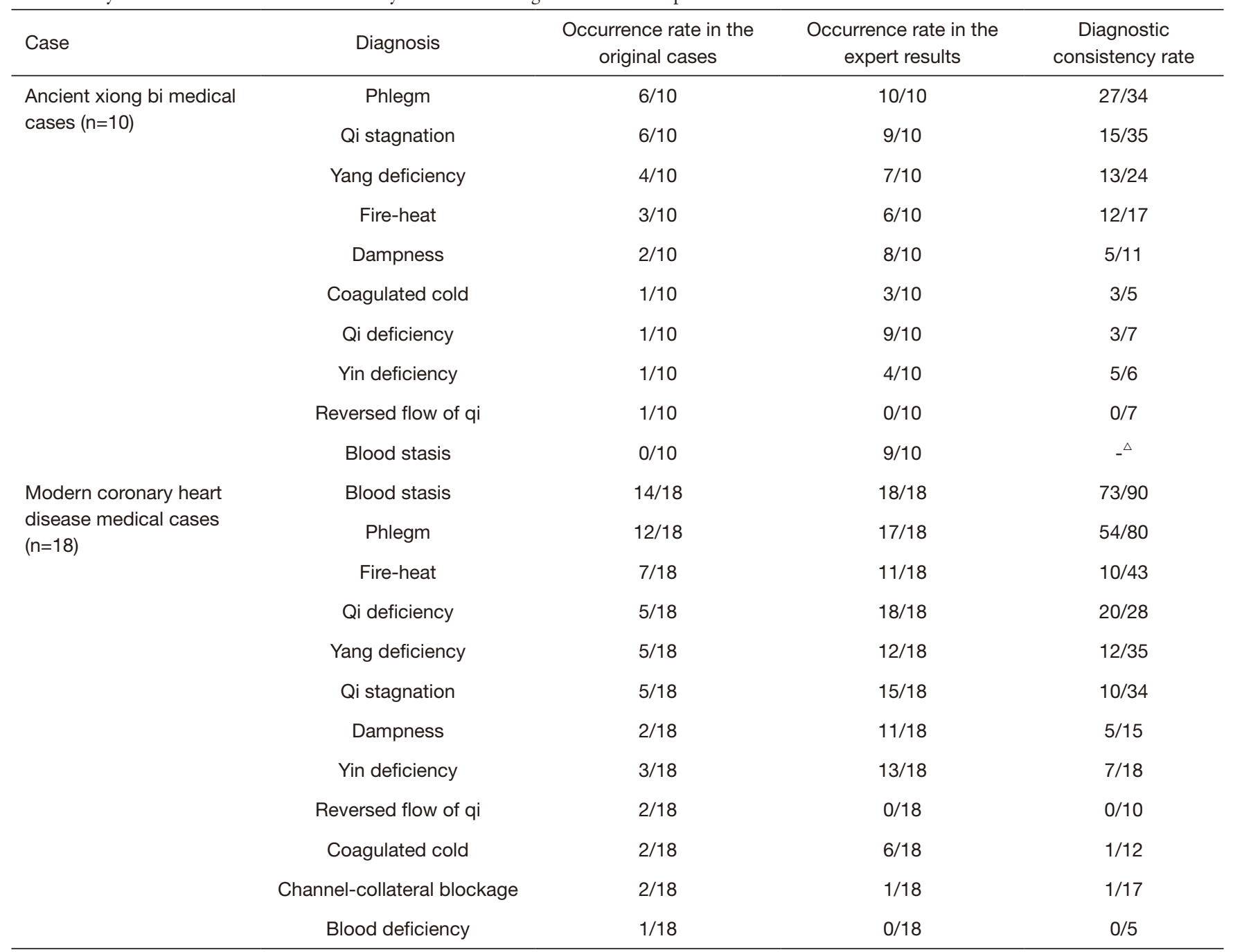

${ }^{\triangle}$, additional diagnosis rate was $27 / 57$.

on tongue or chest pain or dark tongue, and the phlegm syndrome diagnosis based on greasy fur (thin greasy fur or yellow greasy fur) or oppression in chest.

Of the 27 phlegm syndrome diagnostic basics, symptoms together with manifestations of tongue and pulse accounted for $6 / 27$, symptoms and pulse manifestations accounted for $7 / 27$, only tongue manifestations accounted for $6 / 27$, symptoms without manifestations of tongue or pulse accounted for $3 / 27$, symptoms and tongue manifestations accounted for $3 / 27$, only manifestations of pulse accounted for $1 / 27$, and for disease diagnosis, symptoms with pulse manifestations accounted for $1 / 27$. Therefore, the diagnostic basis of "symptoms and/or tongue manifestations" constituted 12/27 (44.4\%) of the total phlegm syndrome diagnostic basics.

Of the 38 blood stasis syndrome diagnostic basics, symptoms and tongue manifestations accounted for $14 / 38$, only tongue manifestations accounted for $7 / 38$, symptoms without manifestations of tongue or pulse accounted for $5 / 38$, symptoms and pulse manifestations accounted for $3 / 38$, symptoms together with manifestations of tongue and pulse accounted for $3 / 38$, manifestations of both pulse and tongue accounted for $2 / 38$, disease diagnosis and symptoms accounted for $1 / 38$ and for disease diagnosis, symptoms and tongue manifestations accounted for $1 / 38$. Therefore, the diagnostic basis of "symptoms and/or tongue manifestations" constituted 28/38 (73.7\%) of the blood stasis syndrome diagnostic basis (see Table 5 for details). 
Table 3 Top 10 frequency of the diagnostic basis for the coronary heart disease with phlegm syndrome

\begin{tabular}{lcc}
\hline No. & Symptom/physical sign & Frequency \\
\hline 1 & Oppression in chest & 33 \\
2 & Slippery pulse & 28 \\
3 & Greasy fur & 24 \\
4 & Coughing of phlegm & 14 \\
5 & Chest pain & 12 \\
6 & Dizziness & 11 \\
7 & White fur & 11 \\
8 & Stringy pulse & 10 \\
9 & Thin fur & 10 \\
10 & Deep pulse & 8 \\
\hline
\end{tabular}

Table 4 Top 10 frequency of the diagnostic basis for the coronary heart disease with blood stasis syndrome

\begin{tabular}{lcc}
\hline No. & Symptom/physical sign & Frequency \\
\hline 1 & Chest pain & 43 \\
2 & Dark tongue & 30 \\
3 & Oppression in the chest & 19 \\
4 & Red tongue & 14 \\
5 & Ecchymosis on the tongue & 13 \\
6 & Stringy pulse & 12 \\
7 & Chest pain involving the back & 8 \\
8 & Dim complexion & 8 \\
9 & Disease of long duration & 8 \\
10 & Backache & 7 \\
\hline
\end{tabular}

${ }^{\diamond}$, specific description of long-term disease of 28 cases: 2 months is the shortest.

\section{Discussion}

\section{Different focus of syndrome differentiation between ancient and modern TCM experts}

In order to compare the difference of CHD syndrome differentiation between ancient and modern TCM experts, this study designed the inclusion and exclusion criteria of ancient CHD medical cases according to the characteristics of the CHD population, pathogenesis and symptoms.
Among the original syndrome diagnosis of the 10 ancient medical cases, syndromes with the high occurrence rate were phlegm syndrome (6/10), qi stagnation syndrome $(6 / 10)$ and yang deficiency syndrome (4/10). Zhang Zhongjing, a well-known physician in the Eastern Han Dynasty, clearly pointed out that "Yang Micro Yin String" was the basic pathogenesis of xiong bi in his book titled Synopsis of the Golden Chamber (12). Yang Micro refers to yang deficiency of upper-energizer and Yin String refers to the cold natured pathogenic factors, such as cold, phlegm, qi stagnation, water and other pathogenic factors invading the upper part of the body $(13,14)$. Later physicians highly praised Zhang Zhongjing's understanding of the pathogenesis of chest discomfort, which mostly identified the pathogenesis of xiong bi from the perspective of cold and phlegm (15). Compared with the ancient doctors, modern TCM experts not only pay attention to the phlegm pathogenesis of patients with xiong bi (or CHD), but also to the blood stasis pathogenesis. In the experts' syndrome differentiation results of the ancient medical cases, the occurrence rate of phlegm and blood stasis syndromes was greater than or equal to $9 / 10$, and the phlegm diagnosis consistency rate was $27 / 34(79.4 \%)$, the additional diagnosis rate of blood stasis was $27 / 57$ (47.4\%). The influence of clinical experience and pathological knowledge of CHD may be the reason why the modern experts prefer to give the blood stasis syndrome diagnosis to patients with xiong bi (or CHD). At present, the main excessive pathogens of CHD have changed from cold coagulation and phlegm to blood stasis and phlegm. In 2011, some scholars (16) analyzed the research literature on TCM syndrome characteristics of CHD published in 1970-2010, and proposed that blood stasis, phlegm and qi stagnation were the most common symptoms of CHD, and the proportions of blood stasis, phlegm and related syndrome types had been increasing. Some scholars (17) had presented statistics on the research literature of TCM syndrome characteristics of CHD published from 2017 to 2018, and found that phlegm syndrome and blood stasis syndrome were still the top two syndromes with values approximately $20 \%$ higher than what published in 2011 (phlegm syndrome from $14.69 \%$ to $36.44 \%$, blood stasis syndrome from $28.10 \%$ to $54.98 \%$ ). In addition, the conclusion of modern research on CHD and blood stasis syndrome may affect the experts' differentiation toward CHD. There are a few same pathological mechanisms between blood stasis syndrome and CHD. Blood stasis refers to the state of stagnation of blood circulation due to qi stagnation, qi deficiency, 
Table 5 Details of experts consultation over 9 coronary heart disease with phlegm-blood stasis syndrome cases

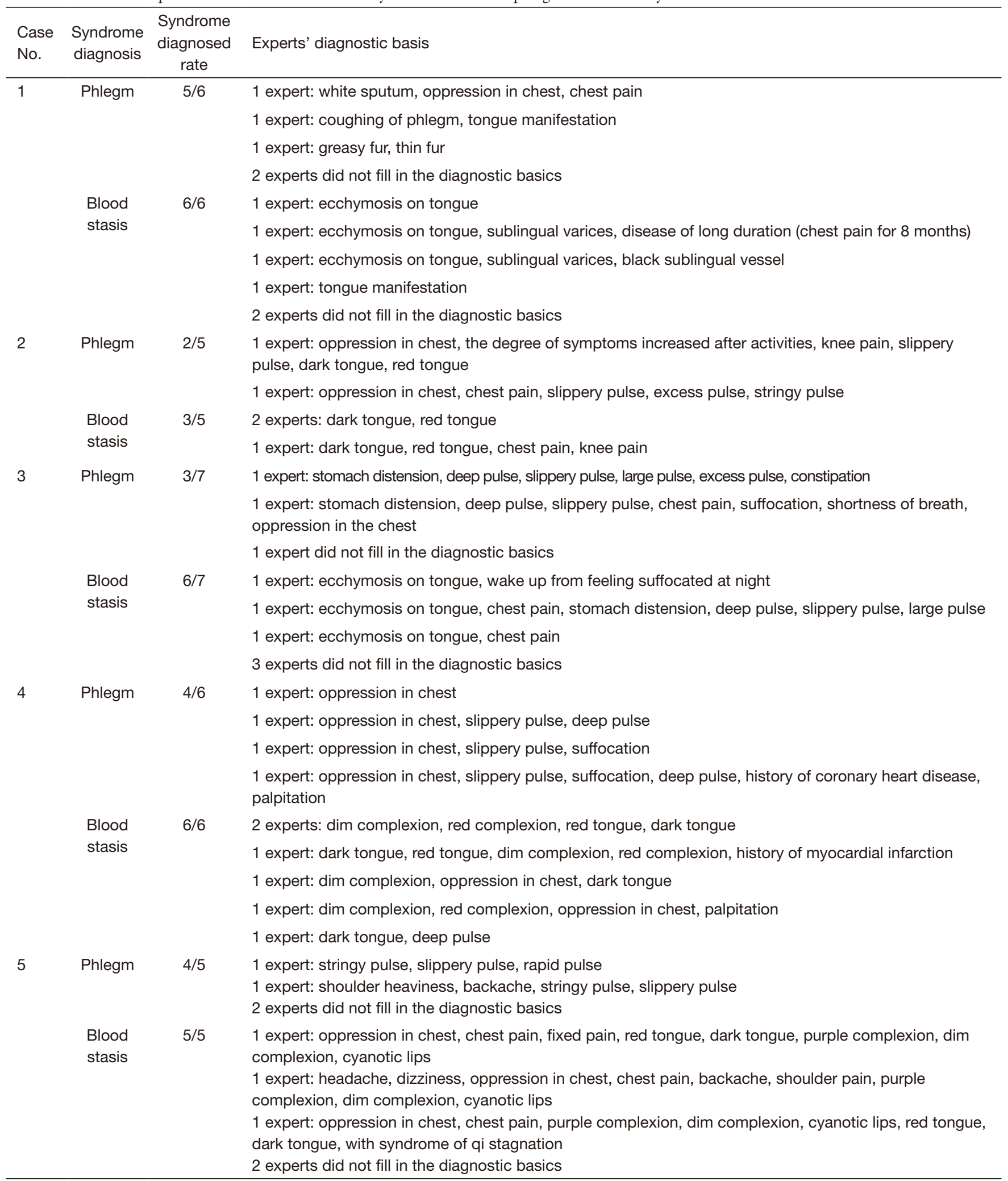

Table 5 (continued) 
Table 5 (continued)

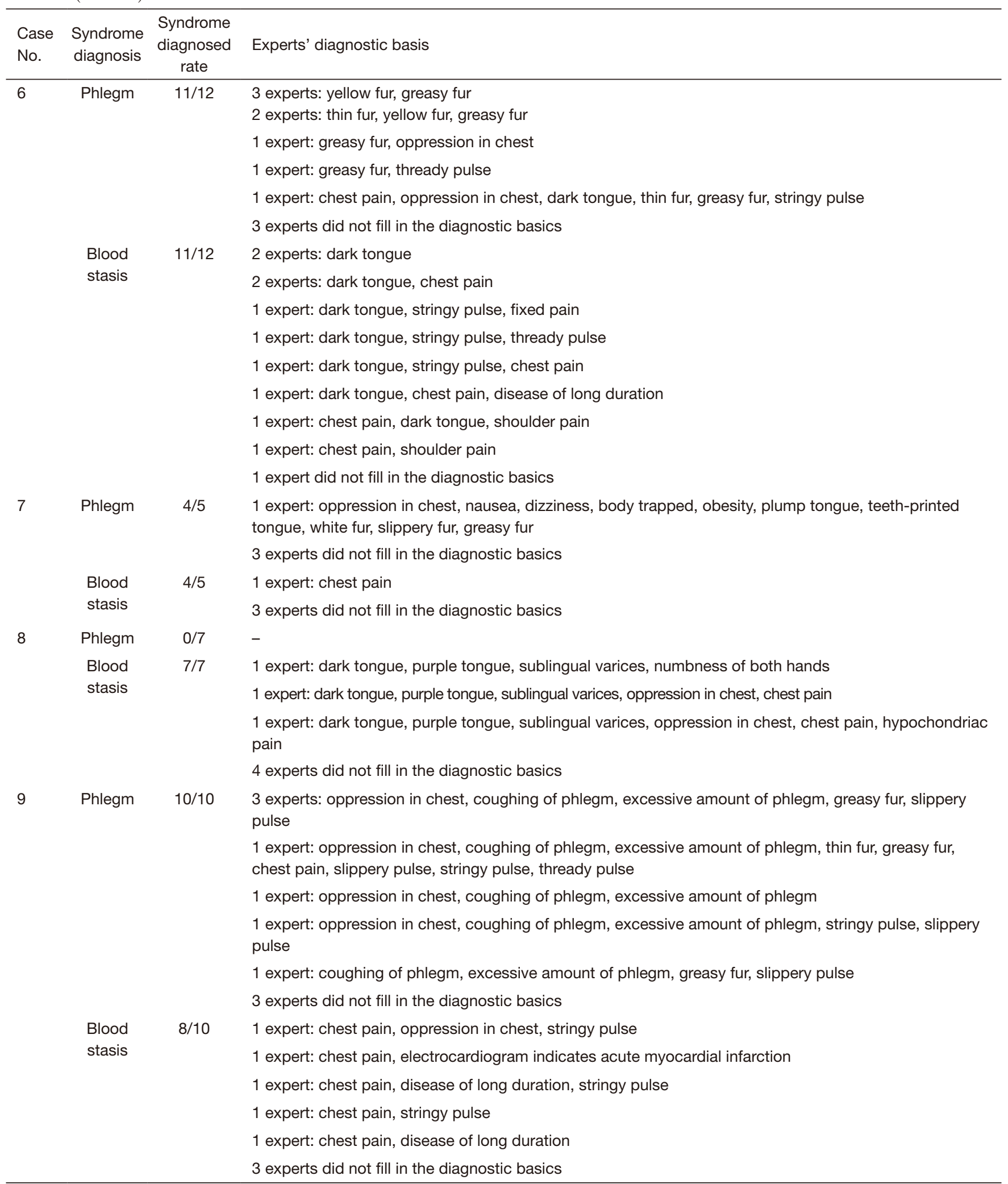


yang deficiency, yin deficiency or external injury (18). Other scholars (19) have explored the essence of blood stasis syndrome from the aspects of microcirculation disorder, abnormal hemodynamics, activating platelets, vascular endothelial damage, inflammatory reaction, etc. The main pathological mechanisms of CHD are coronary artery spasticity, atheromatous plaque instability, activating platelets, and vascular endothelial damage. With the reference of the pathological mechanism and the coordination of the diagnosis and treatment of CHD and blood stasis syndrome, better clinical effects and more research outputs have been achieved. The basic and clinical research on CHD with blood stasis syndrome has become one of the most active and effective fields of academic research of the combination of Chinese and western medicine (20).

\section{Syndrome differentiation strategy for CHD with phlegm and the blood stasis syndromes based on symptoms/physical signs}

Combined with the results and analysis of the experts' diagnostic basics of the phlegm and blood stasis syndromes of CHD cases, it can be seen that the important diagnostic basics of the phlegm syndrome of CHD were oppression in the chest, slippery pulse, greasy fur, coughing of phlegm, stomach distension, etc. The important diagnostic basics of the blood stasis syndrome were dark tongue, ecchymosis on tongue, sublingual varices, chest pain, oppression in the chest, stringy pulse, etc. In the Clinical Diagnosis Standard of Phlegm Dampness Syndrome of Coronary Heart Disease (21) developed and issued by our research group in 2017, the main diagnostic basis included oppression in the chest, slippery pulse, greasy fur, and stomach distension. Within which, greasy fur and slippery pulse are the main indicators. According to the Diagnostic Criteria of Phlegm Syndrome in Traditional Chinese Medicine (22), coughing of phlegm is a secondary symptom of the phlegm syndrome. In the Diagnosis Criteria for Blood Stasis Syndrome of Coronary Heart Disease (23) edited by the Professional Committee of Promoting Blood Circulation and Removing Stasis of Chinese Association of Integrated Traditional and Western Medicine in 2016, the main indicators include dark tongue, ecchymosis on tongue, sublingual varices and chest pain, with sublingual varices as a secondary indicator. It should be pointed out that in the Diagnosis Criteria for Blood Stasis Syndrome of Coronary Heart Disease, chest pain occurring in a fixed position is emphasized. As for stringy pulse, some scholars have proposed that when blood stasis affects the circulation of qi and blood, it may cause the convergence and tension of the two, resulting in a stringy pulse (24). Therefore, stringy pulse is also included as a diagnostic indicator in the Clinical Diagnosis Standard of Phlegm and Blood Stasis Syndrome of Coronary Atherosclerotic Heart Disease (25). To sum up, the important diagnostic indicators of the phlegm and blood stasis syndromes of CHD concluded from this study essentially conform to the current academic understanding of the diagnostic basis of the phlegm and blood stasis syndromes of CHD.

Collecting symptoms and tongue manifestation of patients were the common syndrome differentiation strategy for the phlegm and blood stasis syndromes of CHD of the experts. In the diagnostic basis of these syndromes, as analyzed from the questionnaire, the symptoms or (and) tongue manifestation were the most common diagnostic basics. In this study, the experts referred generally 2-3 diagnostic indicators as the basis for the diagnosis of the phlegm or blood stasis syndrome of CHD. Compared with pulse manifestation, experts could diagnose phlegm syndrome and blood stasis syndrome with only 1 symptom or 1 tongue manifestation. For example, the experts could diagnose the phlegm syndrome based on oppression in the chest or greasy fur, and diagnose the blood stasis syndrome on chest pain, ecchymosis on tongue or dark tongue in patients with CHD. The diagnostic strategy for the phlegm and blood stasis syndromes of the national master of Chinese medicine, professor Zhang JR is paying attention to tongue manifestation, for example, the tongue manifestation of the phlegm syndrome is often greasy fur (white, yellow, sticky or thick greasy fur), while the tongue manifestation of the blood stasis syndrome is often dull purple or, dull red tongue, or with ecchymosis, and sublingual distend, varicose or dark veins (26).

\section{Syndrome differentiation strategy for CHD with the phlegm and blood stasis syndromes based on non- symptoms/non-physical signs}

The results of this study also suggest that experts have not only paid attention to the symptoms and physical signs of the patients, but also to the patients' medical history and the results of modern auxiliary examinations. The history of disease provides doctors with information about the disease and its course. Every disease has its basic pathogenesis. For example, the basic pathogenesis of primary osteoporosis is the deficiency of kidney essence (27); that of functional 
dyspepsia is spleen deficiency, qi stagnation, and stomach disharmony (28), and for constipation, it is disorganized conduction of the large intestine (29). Disease diagnosis suggests the pathogenesis of the disease and provides a guidance for the clinical practice of syndrome differentiation. It reflects the advantages of the combination of disease differentiation and syndrome differentiation. Therefore, the history of disease is of great value for syndrome differentiation. Lei ZY (30), Zhang BL (31,32), Hu JQ (33), Wang J (34) and other experts proposed that phlegm and blood stasis may exist through all phases of CHD. None of the experts diagnosed phlegm syndrome in case No. 8 , though it was noted that "although this patient has been ill for one year and his beart qi was greatly damaged his chronic and serious illness bas not been healed, and the phlegm and blood stasis have not been solved" (35). This suggests that the medical expert diagnosed it as the phlegm and blood stasis syndromes based on the course of disease. In the syndrome differentiation results of case No. 4, one expert diagnosed the phlegm and blood stasis syndromes based on the CHD history of the patient. Therefore, when faced with CHD patients, TCM practitioners should determine whether the patients have the phlegm and blood stasis syndromes first. In addition, a prolonged clinical course is related to blood stasis. The Guide to Clinical Practice with Medical Cases (36) points out that patients with chronic diseases will have blood stasis syndrome. Long-term disease appeared to be a diagnostic basis of blood stasis syndrome as filled in by the experts on the questionnaire.

Progress in science and technology provides new methods for TCM syndrome differentiation. Being fully absorbed by TCM physicians, modern medical technology is actively applied to diagnosis, and thus promotes the objectification and quantification of syndrome differentiation. For example, 2 of the 5 main diagnostic indicators in the Diagnosis Criteria for Blood Stasis Syndrome of Coronary Heart Disease (23) are auxiliary diagnostic indicators, which are coronary angiography showing at least 1 coronary artery stenosis $\geq 75 \%$, and ultrasound or angiography showing coronary artery thrombus or intracardiac mural thrombus. In this study, one expert used the electrocardiogram of myocardial infarction as a diagnostic basis of the blood stasis syndrome. In addition, biochemical test results can be applied to syndrome differentiation of phlegm and blood stasis of CHD, for example, in the Practical Diagnosis Standard of Blood Stasis Syndrome (37), the abnormality of biochemical test results such as hemorheology, coagulation and fibrinolysis are secondary indicators of the blood stasis syndrome diagnosis. Under the guidance of the theory of TCM, auxiliary examinations would enrich the differentiation strategy of clinicians.

\section{Conclusions}

This study summarized the strategy for CHD phlegm and blood stasis syndrome differentiation of modern TCM experts, and found that they paid much attention to the treatment of CHD based on TCM pathology theories of phlegm and blood stasis. Diagnosis based on symptoms and tongue manifestation is the commonly used strategy for CHD phlegm and blood stasis syndrome differentiation. Meanwhile, the medical history and the results of modern auxiliary examinations are also used as an important diagnostic basis of phlegm syndrome and blood stasis syndrome of CHD. Interview can be combined with questionnaire to enrich the conclusions of studies in the future.

Every TCM physician has their individual strategy for clinical syndrome differentiation. The strategy is more than a measure of the doctor's professional ability, but is related to many factors such as personal preferences, knowledge, way of thinking, observation and judgment ability, and practice proficiency. We are looking forward to the further exploration of experts' syndrome differentiation strategy and a simpler and more accurate CHD syndrome differentiation and thus improve the effects of TCM treatment of CHD.

\section{Acknowledgments}

We thank M.M Sheng Zhou from Medical Data Analysis Center of Qingdao Ruyi Software Co., Ltd. and M.M Ke-Gang Li from Anyue County People's Hospital for editorial support.

Funding: This study was supported by the National Basic Research Program of China (973 Program) (No. 2014CB542903), the 11th Batch of Fundamental Research Funds for the Central Public Welfare Research Institutes (No. YZ-1799) and Central Public-Interest Scientific Institution Basal Research Fund of China Academy of Chinese Medical Sciences (No. zz11-104).

\section{Footnote}

Reporting Checklist: The authors have completed the SURGE reporting checklist. Available at https://dx.doi. 
org/10.21037/apm-21-2332

Data Sharing Statement: Available at https://dx.doi. org/10.21037/apm-21-2332

Conflicts of Interest: All authors have completed the ICMJE uniform disclosure form (available at https://dx.doi. org/10.21037/apm-21-2332). The authors have no conflicts of interest to declare.

Ethical Statement: The authors are accountable for all aspects of the work in ensuring that questions related to the accuracy or integrity of any part of the work are appropriately investigated and resolved. The ethical review or the approval of the ethical review committee was not required for this study. The study was conducted in accordance with the Declaration of Helsinki (as revised in 2013). The participants have given informed consent before taking part.

Open Access Statement: This is an Open Access article distributed in accordance with the Creative Commons Attribution-NonCommercial-NoDerivs 4.0 International License (CC BY-NC-ND 4.0), which permits the noncommercial replication and distribution of the article with the strict proviso that no changes or edits are made and the original work is properly cited (including links to both the formal publication through the relevant DOI and the license). See: https://creativecommons.org/licenses/by-nc-nd/4.0/.

\section{References}

1. Timmis A, Townsend N, Gale C, et al. European Society of Cardiology: Cardiovascular Disease Statistics 2017. Eur Heart J 2018;39:508-79.

2. Yang Y, Teng X, Zhu J. Sufentanil blunts the myocardial stress induced by tracheal intubation in older adult patients with coronary heart disease better than equipotent fentanyl. Ann Palliat Med 2020;9:3909-14.

3. Bi YF, Wang XL, Zhao ZQ, et al. Clinical Epidemiological Investigation on Current Syndrome Characteristics of Coronary Heart Disease in Chinese Medicine. Journal of TCM (Chin) 2017;58:2013-9.

4. Wang CC, Xu WM, Jiang LJ, et al. Distribution of Phlegm and Stasis Binding Pattern in Healthy Subjects and All Stages of Coronary Heart Disease: A Multicentered, Cross-sectional Study of 11383 Cases. Journal of Traditional Chinese Medicine (Chin) 2021;62:494-504.
5. Zhao J, Wang Xu, Gao SM, et al. Comparative study on TCM syndrome evaluation scale of phlegm and blood stasis syndrome in coronary heart disease with stable angina pectoris. Tianjin Journal of Traditional Chinese Medicine Jun (Chin) 2020;37:656-60.

6. Xu WM, Yang K, Jiang LJ, et al. Integrated Modules Analysis to Explore the Molecular Mechanisms of PhlegmStasis Cementation Syndrome with Ischemic Heart Disease. Front Physiol 2018;9:7.

7. Hu JQ, Wang CC, Duan F, et al. Study on Macrocosmic Diagnostic Criteria for Coronary Heart Disease with Intermingled Phlegm-Blood Stasis Syndrome. Zhongguo Zhong Xi Yi Jie He Za Zhi 2016;36:1164-8.

8. $\mathrm{Xu}$ WM, Hu JQ, Li JB, et al. Systematic Retrospection and Research Prospects of Contemporary Chinese Medicine Syndrome Differentiation Methods. Journal of Traditional Chinese Medicine (Chin) 2016;57:1531-9.

9. Zhou ZY. Chinese Internal Medicine. Beijing: China Press of Traditional Chinese Medicine, 2017:137.

10. Yao NL. Differential diagnosis of TCM symptoms. 2nd Edition. Beijing: People's Medical Publishing House, 1984:4.

11. Chinese Medicine Term Examination and Approval Committee. Chinese Terms in Traditional Chinese Medicine and Pharmacy. Beijing: China Science Publishing \& Media Ltd., 2005.

12. Tian Y. Discussion on the Meaning of 'Yang Micro Yin String' in the Differentiation and Treatment of Xiong Bi. Liaoning Journal of TCM (Chin) 2012;39:451-2.

13. Zhang XP. New Interpretation of Yang Micro Yin String. Journal of TCM (Chin) 1987;11:68.

14. Yang J, Yuan SY. Guiding Effect of 'Yang Micro Yin String' Theory on Clinical Diagnosis and Treatment of Coronary Heart Disease. Hebei J of TCM (Chin) 2018;40:128-31.

15. Liu R. Research of Ancient Syndrome Differentiation Methods of Thoracic Obstruction. Beijing: China Academy of Chinese Medical Sciences, 2016.

16. Mao JY, Niu ZC, Zhang BL. Literature Analysis of Studies on the TCM Syndromes of Coronary Heart Disease in the Recent 40 Years. Journal of TCM (Chin) 2011;52:958-61.

17. Wang CC, Yang Y, Wu S, et al. Study on the Evolution Rule of TCM Syndromes in Different Development Stages of Coronary Heart Disease. CJTCMP (Chin) 2019;34:2101-6.

18. Lu HR, Hu JQ. Analysis of 'Static Blood' and 'Blood Stasis'. CJTCMP (Chin) 2017;32:426-8.

19. Fu CG. Establishment and Evolution of Modern Blood Stasis Syndrome. Shandong Journal of TCM (Chin) 2016;35:1081-4, 1088. 
20. Zhang J, Chen KJ, Tian JZ. On the Formation and Development of the Theory of Blood Stasis Syndrome. Journal of Hubei College of TCM (Chin) 2005;7:39-40.

21. Hu JQ, Xu WM, Wang CC, et al. Interpretation of Clinical Diagnosis Standard of Phlegm Dampness Syndrome of Coronary Heart Disease. Journal of Basic Chinese Medicine (Chin) 2017;23:1247-52.

22. Wu HL, Lv WH, Pan GJ, et al. Diagnostic Criteria of Phlegm Syndrome in Traditional Chinese Medicine. CJITWM (Chin) 2016;36:776-80.

23. Professional Committee for Promoting Blood Circulation and Removing Blood Stasis of Chinese Association of Integrative Medicine. Diagnostic Criteria for Blood Stasis Syndrome of Coronary Heart Disease. CJITWM (Chin) 2016;36:1162.

24. Jiang LJ, Hu JQ, Li RJ, et al. Pulse Manifestations and Influence Factors of Coronary Heart Disease with Intermingled Phlegm-Blood Stasis Syndrome. Global Traditional Chinese Medicine (Chin) 2015;8:641-5.

25. China Association of Chinese Medicine. Clinical Diagnosis Standard of Phlegm and Blood Stasis Syndrome of Coronary Atherosclerotic Heart Disease. Beijing: China Press of Traditional Chinese Medicine, 2018.

26. Zhang CJ, Wang SP, Zhang JR. Zhang Jingren's Clinical Experience of Treating Phlegm and Blood Stasis. Shandong Journal of TCM (Chin) 2008;27:418-9.

27. Ge JR, Zheng HX, Wan XM, et al. Expert Consensus on the Prevention and Treatment for Primary Osteoporosis with Traditional Chinese Medicine. Chin J Osteoporosis (Chin) 2015;21:1023-8.

28. Spleen and Stomach Disease Branch of China Association of Chinese Medicine. Expert Consensus on TCM Diagnosis and Treatment of Functional Dyspepsia (2017). CJTCMP (Chin) 2017;32:2595-8.

29. Spleen and Stomach Disease Branch of China Association of Chinese Medicine. Expert Consensus on TCM

Cite this article as: Liu R, Jiang LJ, Yang Y, Wang CC, Tong X, Xu WM, Wu M, Lu KZ, Hu JQ. Study on syndrome differentiation strategy of phlegm and blood stasis syndromes of coronary heart disease based on expert consultation on medical cases. Ann Palliat Med 2021;10(9):9940-9952. doi: 10.21037/ apm-21-2332
Diagnosis and Treatment of Constipation (2017). Journal of TCM (Chin) 2017;58:1345-50.

30. Wu XP, Yu XY, Liu CF, et al. Chief Physician Lei Zhongyi's Experience of Treating Angina Pectoris with Phlegm, Blood Stasis and Toxin. Shaanxi Zhongyi (Chin) 2010;31:1507-8.

31. Zhang H, Kang LY, Zhang BL. Analysis of Phlegm and Blood Stasis Syndrome in Cardiovascular and Cerebrovascular Diseases. Tianjin J of TCM (Chin) 2009;26:172-4.

32. Li B, Mao JY, Jiang F, et al. Zhang Boli's Examples of Paired Drugs Application in the Treatment of Coronary Heart Disease with Phlegm and Blood Stasis Syndrome. Journal of TCM (Chin) 2013;54:910-2.

33. Cai YR, Jiang LJ, Li ZY, et al. Accompany and Transformation of Phlegm and Blood Stasis: Theory and Clinical Experience in The Treatment of Coronary Heart Disease. Journal of Basic Chinese Medicine (Chin) 2019;25:100-2, 126.

34. Wang J, Xing YW, Yao KW, et al. Connotation and Extension of the Theory of Phlegm-Blood StasisStagnation-Deficiency in Coronary Heart Disease. Journal of TCM (Chin) 2019;60:280-4.

35. Zhao YY, Ren BQ. Professor Ren Jixue's Experience in the Diagnosis and Treatment of Real Heartache (Myocardial Infarction). Journal of Hubei University for Nationalities (Medical Edition) (Chin) 2010;27:49-50.

36. Ye G. Guide to Clinical Practice with Medical Cases. Shanxi: Shanxi Science and Technology Press, 2006:393.

37. Professional Committee for Promoting Blood Circulation and Removing Blood Stasis of Chinese Association of Integrative Medicine. Practical Diagnosis Standard of Blood Stasis Syndrome. CJITWM (Chin) 2016;36:1163.

(English Language Editor: K. Brown) 